\title{
Study on Synthesis and Biological Activity of Some Pyridopyridazine Derivatives
}

\author{
Sevilay Akçay, ${ }^{1}$ Mahmut Ülger, ${ }^{2}$ Fatma Kaynak Onurdag ${ }^{3}$ \\ and Yasemin Dündar ${ }^{1}{ }^{\star}$ \\ ${ }^{1}$ Department of Pharmaceutical Chemistry, Faculty of Pharmacy, Gazi University, Ankara, Turkey. \\ ${ }^{2}$ Department of Pharmaceutical Microbiology, Faculty of Pharmacy, Mersin University, Mersin, Turkey. \\ ${ }^{3}$ Department of Pharmaceutical Microbiology, Faculty of Pharmacy, Trakya University, Edirne, Turkey. \\ *Corresponding author: E-mail: yasemina@gazi.edu.tr, akkocysmn@gmail.com \\ Phone: +90-312-2023237; Fax: +90-312-2235018
}

Received: 06-07-2018

\begin{abstract}
In this study, new pyrido[3,4- $d$ ]pyridazine derivatives were synthesized and evaluated for their in vitro antibacterial, antifungal and antimy cobacterial activities. Among the synthesized compounds, compound 10 (1-(4-benzylpiperazin-1-yl) pyrido[3,4-d]pyridazin-4(3H)-one) and compound 12 (1-(4-benzylpiperidin-1-yl)pyrido[3,4-d]pyridazin-4(3H)-one) were found to have the highest antimycobacterial activity. However, all compounds were found ineffective against tested Gram-positive, Gram-negative bacteria and fungus.
\end{abstract}

Keywords: Pyrido[3,4- $d]$ pyridazine derivatives; pyrido[3,4- $d]$ pyridazin-4(3H)-one; pyrido[3,4- $d]$ pyridazin- $1(2 H)$-one; antimycobacterial activity.

\section{Introduction}

Tuberculosis (TB) is a chronic and often deadly infectious disease caused by the Mycobacterium tuberculosis. ${ }^{1}$ According to the World Health Organization (WHO) Global Tuberculosis Report 2017, in 2016, there were an estimated 1.3 million TB deaths among HIV-negative people and an additional 374.000 deaths among HIV-positive people. ${ }^{1}$ Standard TB therapy involves taking isoniazid (INH), rifampicin (RIF), pyrazinamide (PZA) and ethambutol (EMB) for two months (intensive phase), prolong treatment with INH and RIF for four months (continuation phase). ${ }^{2}$ In patients with RIF-resistant TB or multidrug-resistant $\mathrm{TB}$, treatment regimens with at least five effective antituberculosis agents during the intensive phase is recommended, including PZA and four group second-line antituberculosis drugs. The regimen may be further strengthened with high dose INH and/or EMB. ${ }^{3}$

INH, the first-line drug, is a key component in all TB therapy regimens recommended by the WHO. ${ }^{1-3} \mathrm{PZA}$ is also an important first-line drug which has sterilizing activity against semi-dormant tuberculin bacilli. ${ }^{4}$ Hence, re- searchers have modified the INH and PZA scaffolds to develop novel compounds to obtain better antitubercular activity. Additionally, many researchers have worked on hybrid molecules of INH and other antitubercular drugs. ${ }^{5-12}$ Therefore, our group decided to study pyrido[3,4- $d]$ pyridazine ring system which has INH and PZA like scaffold (Figure 1).<smiles>NNC(=O)c1ccncc1</smiles>

$\mathrm{INH}$<smiles>NC(=O)c1cnccn1</smiles>

PZA<smiles>O=c1[nH][nH]c(=O)c2cnccc12</smiles>

(1)<smiles>[R]c1n[nH]c(=O)c2ccncc12</smiles>

$(3,5-8)$<smiles>[R]c1n[nH]c(=O)c2cnccc12</smiles>

$(4,9-12)$
Figure 1. INH, PZA and the general structure of the synthesized compounds. 
2,3-Dihydropyrido[3,4-d]pyridazin-1,4-dione

and its derivatives are generally synthesized by the reaction of hydrazine hydrate with 3,4-pyridinedicarboxylic acid derivatives, such as 3,4-pyridinedicarboxylic anhydride, 3,4-pyridinedicarbonitriles, dimethyl or diethyl pyridine-3,4-dicarboxylate, $1 H$-pyrrolo[3,4-c]pyridine- 1,3 $(2 \mathrm{H})$-dione. ${ }^{13-17}$ In this study, 3,4-pyridinedicarboxylic acid was used as the starting material and then converted to 3,4-pyridinedicarboxylic anhydride via acetic acid anhydride, followed by the cyclization with hydrazine hydrate. Subsequent to chlorination of pyridazinone ring with phosphorus oxychloride and hydrolysis to monochloro derivatives, the final compounds were obtained via nucleophilic aromatic substitution reaction. Synthesized novel 4 -substituted pyrido[3,4- $d]$ pyridazin-1 $(2 H)$-one derivatives and 1-substituted pyrido[3,4- $d]$ pyridazin-4(3H)-one derivatives were evaluated for their antimycobacterial, antibacterial, and antifungal properties.

\section{Experimental}

\section{1. Chemistry}

All chemicals and solvents were purchased locally from Merck AG and Aldrich Chemicals. The microwave reaction was carried out in a MicroSYNTH Microwave Lab station (Milestone S.r.l.). Flash chromatography was performed with a Combi-flashRf automated flash chromatography system with RediSep columns (Teledyne-Isco, Lincoln, NE, USA). Melting points were determined with an Electrothermal-9200 Digital Melting Point Apparatus and are uncorrected. Fourier-transform infrared attenuated total reflectance (FTIR-ATR) spectra were recorded on Perkin Elmer Spectrum 400 FT-IR and FT-NIR spectrometers with a Universal ATR sampler. ${ }^{1} \mathrm{H}$ NMR spectra were recorded in DMSO- $d_{6}$ on a Varian Mercury $400,400 \mathrm{MHz}$ High Performance Digital FT-NMR spectrometer at the NMR facility of the Faculty of Pharmacy, Ankara University. All chemical shifts were recorded as $\delta$ (ppm). Microanalyses for $\mathrm{C}, \mathrm{H}$, and $\mathrm{N}$ were performed on a Leco-932 at the Faculty of Pharmacy, Ankara University, Ankara, Turkey, and they were within the range of $\pm 0.4 \%$ of the theoretical value. HRMS spectra were taken on a Waters LCT Premier XE orthogonal acceleration time-of-flight (oaTOF) mass spectrometer using ESI (+) or ESI (-) methods (Waters Corporation, Milford, MA, USA). The syntheses of 2,3-dihydropyrido[3,4- $d]$ pyridazin-1,4-dione (compound 1), ${ }^{13}$ 1,4-dichloropyrido[3,4-d]pyridazine (compound 2), ${ }^{13}$ 4-chloropyrido[3,4-d]pyridazin-1(2H)-one (compound 3), ${ }^{13,14}$ 1-chloropyrido[3,4-d]pyridazin-4(3H)one (compound 4$)^{13,14}$ were previously reported.

\section{2,3-Dihydropyrido[3,4-d]pyridazin-1,4-dione (1)}

$8.61 \mathrm{~g}$ (0.05 mol) 3,4-pyridinedicarboxylic acid was added to $30 \mathrm{~mL}(0.31 \mathrm{~mol})$ acetic acid anhydride, and the mixture was heated to reflux and stirred for $1 \mathrm{~h}$. After cool- ing to room temperature, $30 \mathrm{~mL}$ hydrazine hydrate was added and the mixture was refluxed and stirred for $4 \mathrm{~h}$. The product was collected by suction filtration, washed with water and dried. Crystallized from ethanol/water to yield 98.6\%; mp $>300{ }^{\circ} \mathrm{C}$; FTIR-ATR: $3404(\mathrm{O}-\mathrm{H}), 3300-$ $2200(\mathrm{~N}-\mathrm{H}), 1668(\mathrm{C}=\mathrm{O}) \mathrm{cm}^{-1} ;{ }^{1} \mathrm{H}$ NMR (DMSO- $\left.d_{6}\right): \delta$ $9.34(1 \mathrm{H}, \mathrm{s}, \mathrm{H} 5), 9.03\left(1 \mathrm{H}, \mathrm{d}, J_{7-8}=5.6 \mathrm{~Hz}, \mathrm{H} 7\right), 7.90$ $\left(1 \mathrm{H}, \mathrm{d}, J_{8-7}=5.6 \mathrm{~Hz}, \mathrm{H} 8\right)$; HRMS calcd. for $\mathrm{C}_{7} \mathrm{H}_{6} \mathrm{~N}_{3} \mathrm{O}_{2}$ $[\mathrm{M}-\mathrm{H}]^{+}$: 164.0460. Found: 164.0455. Anal. Calcd. for $\mathrm{C}_{7} \mathrm{H}_{5} \mathrm{~N}_{3} \mathrm{O}_{2} \cdot 1 / 3 \mathrm{H}_{2} \mathrm{O}: \mathrm{C}, 49.71 ; \mathrm{H}, 3.38 ; \mathrm{N}, 24.84$. Found: C, 49.78; H, 3.27; N, 25.06\%.

\section{1,4-Dichloropyrido[3,4-d]pyridazine (2)}

A mixture of $6.52 \mathrm{~g}$ (0.04 mol) 2,3-dihydropyrido[3,4-d]pyridazin-1,4-dione and $18.6 \mathrm{~mL}(0.19 \mathrm{~mol})$ phosphorus oxychloride in $6.46 \mathrm{~mL}(0.08 \mathrm{~mol})$ pyridine was heated to reflux and stirred for $5 \mathrm{~h}$. The mixture then was poured into a slush of $200 \mathrm{~g}$ of ice and then neutralized with $\mathrm{NaHCO}_{3}$ and then extracted with $3 \times 100 \mathrm{~mL}$ ethyl acetate. Organic phase was separated, washed with water, dried with $\mathrm{Na}_{2} \mathrm{SO}_{4}$ and evaporated to dryness (yield 45\%). 1,4-Dichloropyrido[3,4- $d]$ pyridazine was subsequently used without further purification.

\section{4-Chloropyrido[3,4- $d]$ pyridazin-1(2H)-one (3) and} 1-Chloropyrido[3,4-d]pyridazin-4(3H)-one (4)

1,4-Dichloropyrido[3,4- $d]$ pyridazine $(5 \mathrm{~g}, 0.025 \mathrm{~mol})$ was heated up to reflux temperature in $150 \mathrm{~mL}$ diluted $\mathrm{HCl}$ (1\%) for $2 \mathrm{~h}$. After cooling, the precipitate formed was filtered off, dried and crystallized from acetic acid to obtain 4-chloropyrido[3,4-d]pyridazin-1 $(2 H)$-one with yield of $52 \%$. The remaining reaction medium was neutralized with ammonium hydroxide to precipitate the 1-chloropyrido[3,4-d]pyridazin-4(3H)-one, which was isolated by filtration, washed with water, dried and crystallized from water (yield 34\%).

\section{4-Chloropyrido[3,4-d]pyridazin-1(2H)-one (3)}

Crystallized from acetic acid to yield $52 \% ; \mathrm{mp} 236^{\circ} \mathrm{C}$; FTIR-ATR: 3220-2400 (N-H), $1678(\mathrm{C}=\mathrm{O}) \mathrm{cm}^{-1}$; ${ }^{1} \mathrm{H}$ NMR $\left(\mathrm{DMSO}-d_{6}\right): \delta 13.14(1 \mathrm{H}, \mathrm{s}, \mathrm{NH}), 9.32\left(1 \mathrm{H}, \mathrm{d}, J_{5-8}=0.8 \mathrm{~Hz}\right.$, H5), $9.10\left(1 \mathrm{H}, \mathrm{d}, J_{7-8}=4.8 \mathrm{~Hz}, \mathrm{H} 7\right), 8.11\left(1 \mathrm{H}, \mathrm{dd}, J_{8-7}=5.2\right.$ $\left.\mathrm{Hz}, J_{8-5}=0.8 \mathrm{~Hz}, \mathrm{H} 8\right)$; HRMS calcd. for $\mathrm{C}_{7} \mathrm{H}_{5} \mathrm{ClN}_{3} \mathrm{O}$ $[\mathrm{M}-\mathrm{H}]^{+}$: 182.0121. Found 182.0116. Anal. calcd. for $\mathrm{C}_{7} \mathrm{H}_{4} \mathrm{ClN}_{3} \mathrm{O}$ : C, 46.30; $\mathrm{H}, 2.22 ; \mathrm{N}, 23.14$. Found: C, 46.11; $\mathrm{H}, 2.22 ; \mathrm{N}, 23.04 \%$.

\section{1-Chloropyrido[3,4-d]pyridazin-4(3H)-one (4)}

Crystallized from water to yield $34 \%$; mp $172{ }^{\circ} \mathrm{C}$; FTIR-ATR: 3400-2400 (N-H), $1682(\mathrm{C}=\mathrm{O}) \mathrm{cm}^{-1} ;{ }^{1} \mathrm{H}$ NMR (DMSO- $\left.d_{6}\right): \delta 13.13(1 \mathrm{H}, \mathrm{s}, \mathrm{NH}), 9.45(1 \mathrm{H}, \mathrm{s}, \mathrm{H} 5)$, $9.13\left(1 \mathrm{H}, \mathrm{d}, J_{7-8}=5.2 \mathrm{~Hz}, \mathrm{H} 7\right), 7.84\left(1 \mathrm{H}, \mathrm{dd}, J_{8-7}=5.6 \mathrm{~Hz}\right.$, $J_{8-5}=0.8 \mathrm{~Hz}, \mathrm{H} 8$ ); HRMS calcd. for $\mathrm{C}_{7} \mathrm{H}_{5} \mathrm{ClN}_{3} \mathrm{O}[\mathrm{M}-\mathrm{H}]^{+}$: 182.0121. Found 182.0114. Anal. calcd. for $\mathrm{C}_{7} \mathrm{H}_{4} \mathrm{ClN}_{3} \mathrm{O}: \mathrm{C}$, 46.30; H, 2.22; N, 23.14. Found: C, 46.12; H, 2.28; N, $23.23 \%$. 
General Procedure for the Synthesis of 4-Substituted Pyrido $[3,4-d]$ pyridazin-1 $(2 H)$-one Derivatives or 1-Substituted Pyrido[3,4- $d]$ pyridazin-4(3H)-one Derivatives

0.003 mol 4-chloropyrido[3,4- $d]$ pyridazin-1(2H)one or 1-chloropyrido[3,4-d]pyridazin-4(3H)-one in $5 \mathrm{~mL}$ diethylene glycol was treated with $0.015 \mathrm{~mol}$ piperazine or piperidine derivatives. The reaction mixture was heated to $140{ }^{\circ} \mathrm{C}$ (only exception compound 11: $125^{\circ} \mathrm{C}$ ) for appropriate time under microwave irradiation. Reaction mixture was poured into ice-water mixture and then extracted with $\mathrm{CH}_{2} \mathrm{Cl}_{2}$. Organic phase was separated, washed with water, dried with $\mathrm{Na}_{2} \mathrm{SO}_{4}$, and evaporated to dryness and the crude product thus obtained was purified by flash chromatography.

\section{4-(4-Methylpiperazin-1-yl)pyrido[3,4-d]pyridazin- 1(2H) -one (5)}

Reaction time: $120 \mathrm{~min}$. The crude product was purified by column chromatography using dichloromethane-methanol gradient system. Yield: $17.94 \%$; mp $199{ }^{\circ} \mathrm{C}$; FTIR-ATR: 3330-2400 (N-H), $1678 \quad(\mathrm{C}=\mathrm{O}) \quad \mathrm{cm}^{-1}$; ${ }^{1} \mathrm{H}-\mathrm{NMR}\left(\mathrm{DMSO}-d_{6}\right): \delta 12.43(1 \mathrm{H}, \mathrm{s}, \mathrm{NH}), 9.22(1 \mathrm{H}, \mathrm{s}$, $\mathrm{H} 5), 8.97\left(1 \mathrm{H}, \mathrm{d}, J_{7-8}=4.8 \mathrm{~Hz}, \mathrm{H} 7\right), 8.06\left(1 \mathrm{H}, \mathrm{d}, J_{8-7}=4.8\right.$ $\mathrm{Hz}, \mathrm{H} 8), 3.14$ (4H, m, piperazine $\mathrm{H} 2, \mathrm{H} 6), 2.58$ (4H, m, piperazine $\mathrm{H} 3, \mathrm{H} 5), 2.27\left(3 \mathrm{H}, \mathrm{s}, \mathrm{CH}_{3}\right)$; HRMS calcd. for $\mathrm{C}_{12} \mathrm{H}_{16} \mathrm{~N}_{5} \mathrm{O}[\mathrm{M}-\mathrm{H}]^{+}: 246.1355$. Found 246.1351. Anal. calcd. for $\mathrm{C}_{12} \mathrm{H}_{15} \mathrm{~N}_{5} \mathrm{O} \cdot 1 / 3 \mathrm{H}_{2} \mathrm{O}$ : C, 57.36; H, 6.28; N, 27.87 . Found: C, 57.69; H, 5.93; N, 27.53\%.

\section{4-(4-Benzylpiperazin-1-yl)pyrido[3,4-d]pyridazin-1(2H) -one (6)}

Reaction time: $60 \mathrm{~min}$. The crude product was purified by column chromatography using dichloromethane-methanol gradient system. The collected product was crystallized from ethanol to yield $23.75 \%$; mp $245{ }^{\circ} \mathrm{C}$; FTIR-ATR: 3200-2700 (N-H), $1656(\mathrm{C}=\mathrm{O}) \mathrm{cm}^{-1} ;{ }^{1} \mathrm{H}$ NMR (DMSO- $\left.d_{6}\right): \delta 12.42(1 \mathrm{H}, \mathrm{s}, \mathrm{NH}), 9.22(1 \mathrm{H}, \mathrm{s}, \mathrm{H} 5)$, $8.97\left(1 \mathrm{H}, \mathrm{d}, J_{7-8}=5.2 \mathrm{~Hz}, \mathrm{H} 7\right), 8.06\left(1 \mathrm{H}, \mathrm{dd}, J_{8-7}=4.8 \mathrm{~Hz}\right.$, $\left.J_{8-5}=0.8 \mathrm{~Hz}, \mathrm{H} 8\right), 7.35-7.27\left(5 \mathrm{H}, \mathrm{m}, \mathrm{C}_{6} \mathrm{H}_{5}\right), 3.58(2 \mathrm{H}$, s, $\left.\mathrm{CH}_{2}\right), 3.15(4 \mathrm{H}, \mathrm{m}$, piperazine $\mathrm{H} 2, \mathrm{H} 6), 2.62(4 \mathrm{H}, \mathrm{m}$, piperazine $\mathrm{H} 3, \mathrm{H} 5)$; HRMS calcd. for $\mathrm{C}_{18} \mathrm{H}_{20} \mathrm{~N}_{5} \mathrm{O}[\mathrm{M}-\mathrm{H}]^{+}$: 322.1668. Found 322.1667. Anal. calcd. for $\mathrm{C}_{18} \mathrm{H}_{19} \mathrm{~N}_{5} \mathrm{O} \cdot 1 / 5 \mathrm{H}_{2} \mathrm{O}: \mathrm{C}, 66.53 ; \mathrm{H}, 6.02 ; \mathrm{N}, 21.55$. Found: $\mathrm{C}$, $66.82 ; \mathrm{H}, 5.96 ; \mathrm{N}, 21.53 \%$.

\section{4-(4-Methylpiperidin-1-yl)pyrido[3,4-d]pyridazin-1(2H) -one (7)}

Reaction time: $60 \mathrm{~min}$. The crude product was purified by column chromatography using ethyl acetate. The collected product was crystallized from ethanol to yield 40.93\%; mp $218{ }^{\circ} \mathrm{C}$; FTIR-ATR: 3300-2700 (N-H), 1669 $(\mathrm{C}=\mathrm{O}) \mathrm{cm}^{-1} ;{ }^{1} \mathrm{H}$ NMR (DMSO- $\left.d_{6}\right): \delta 12.37(1 \mathrm{H}, \mathrm{s}, \mathrm{NH})$, $9.19(1 \mathrm{H}, \mathrm{s}, \mathrm{H} 5), 8.96\left(1 \mathrm{H}, \mathrm{d}, J_{7-8}=5.2 \mathrm{~Hz}, \mathrm{H} 7\right), 8.05(1 \mathrm{H}$, $\left.\mathrm{dd}, J_{8-7}=5.2 \mathrm{~Hz}, J_{8-5}=0.8 \mathrm{~Hz}, \mathrm{H} 8\right), 3.47-3.44(2 \mathrm{H}, \mathrm{m}$, piperidine $\mathrm{H} 2, \mathrm{H} 6), 2.76(2 \mathrm{H}$, td, piperidine $\mathrm{H} 2, \mathrm{H} 6)$, 1.761.72 (2H, m, piperidine $\mathrm{H} 3, \mathrm{H} 5), 1.57-1.54$ (1H, m, piperi- dine $\mathrm{H} 4), 1.45(2 \mathrm{H}, \mathrm{m}$, piperidine $\mathrm{H} 3, \mathrm{H} 5), 0.99(3 \mathrm{H}, \mathrm{d}$, $\mathrm{CH}_{3}$ ); HRMS calcd. for $\mathrm{C}_{13} \mathrm{H}_{17} \mathrm{~N}_{4} \mathrm{O}[\mathrm{M}-\mathrm{H}]^{+}$: 245.1402 . Found 245.1407. Anal. calcd. for $\mathrm{C}_{13} \mathrm{H}_{16} \mathrm{~N}_{4} \mathrm{O} \cdot 1 / 5 \mathrm{H}_{2} \mathrm{O}$ : C, 62.99; H, 6.67; N, 22.60. Found: C, 63.26; H, 6.49; N, 22.53\%.

\section{4-(4-Benzylpiperidin-1-yl)pyrido[3,4-d]pyridazin-1(2H) -one (8)}

Reaction time: $30 \mathrm{~min}$. The crude product was purified by column chromatography using $n$-hexane-ethyl acetate gradient system. The collected product was crystallized from ethanol to yield $18.42 \%$; $\mathrm{mp} 225^{\circ} \mathrm{C}$; FTIR-ATR: 3300-2700 (N-H), $1685 \quad(\mathrm{C}=\mathrm{O}) \quad \mathrm{cm}^{-1} ; \quad{ }^{1} \mathrm{H} \quad \mathrm{NMR}$ $\left(\mathrm{DMSO}-d_{6}\right): \delta 12.37(1 \mathrm{H}, \mathrm{s}, \mathrm{NH}), 9.18(1 \mathrm{H}, \mathrm{s}, \mathrm{H} 5), 8.96$ $\left(1 \mathrm{H}, \mathrm{d}, J_{7-8}=5.6 \mathrm{~Hz}, \mathrm{H} 7\right), 8.05\left(1 \mathrm{H}, \mathrm{d}, J_{8-7}=5.2 \mathrm{~Hz}, \mathrm{H} 8\right)$, 7.32-7.19 (5H, m, $\left.\mathrm{C}_{6} \mathrm{H}_{5}\right), 3.49-3.46(2 \mathrm{H}, \mathrm{m}$, piperidine $\mathrm{H} 2, \mathrm{H} 6), 2.74-2.68(2 \mathrm{H}, \mathrm{m}$, piperidine $\mathrm{H} 2, \mathrm{H} 6), 2.61(2 \mathrm{H}$, d, $\left.\mathrm{CH}_{2}\right), 1.72-1.69(3 \mathrm{H}, \mathrm{m}$, piperidine $\mathrm{H} 3, \mathrm{H} 5, \mathrm{H} 4), 1.51-$ $1.49(2 \mathrm{H}, \mathrm{m}$, piperidine $\mathrm{H} 3, \mathrm{H} 5)$; HRMS calcd. for $\mathrm{C}_{19} \mathrm{H}-$ ${ }_{21} \mathrm{~N}_{4} \mathrm{O}[\mathrm{M}-\mathrm{H}]^{+}$: 321.1715. Found 321.1708. Anal. calcd. for $\mathrm{C}_{19} \mathrm{H}_{20} \mathrm{~N}_{4} \mathrm{O} \cdot 1 / 2 \mathrm{C}_{2} \mathrm{H}_{5} \mathrm{OH}$ : C, 69.95; H, 6.75; N, 16.31 . Found: C, 69.78; H, 6.54; N, 16.53\%.

\section{1-(4-Methylpiperazin-1-yl)pyrido[3,4-d]pyridazin-4(3H) -one (9)}

Reaction time: $60 \mathrm{~min}$. The crude product was purified by column chromatography using dichloromethane-methanol gradient system. The collected product was crystallized from ethanol to yield 20.38\%; mp $206{ }^{\circ} \mathrm{C}$; FTIR-ATR: $3300-2600(\mathrm{~N}-\mathrm{H}), 1670(\mathrm{C}=\mathrm{O}) \mathrm{cm}^{-1} ;{ }^{1} \mathrm{H}$ NMR (DMSO- $\left.d_{6}\right): \delta 12.41(1 \mathrm{H}, \mathrm{s}, \mathrm{NH}), 9.42(1 \mathrm{H}, \mathrm{s}, \mathrm{H} 5)$, $9.01\left(1 \mathrm{H}, \mathrm{d}, J_{7-8}=5.2 \mathrm{~Hz}, \mathrm{H} 7\right), 7.75\left(1 \mathrm{H}, \mathrm{d}, J_{8-7}=5.6\right.$ $\mathrm{Hz}, \mathrm{H} 8), 3.09$ (4H, m, piperazine $\mathrm{H} 2, \mathrm{H} 6), 2.55$ (4H, m, piperazine $\mathrm{H} 3, \mathrm{H} 5), 2.26\left(3 \mathrm{H}, \mathrm{s}, \mathrm{CH}_{3}\right)$; HRMS calcd. for $\mathrm{C}_{12} \mathrm{H}_{16} \mathrm{~N}_{5} \mathrm{O}[\mathrm{M}-\mathrm{H}]^{+}: 246.1355$. Found 246.1360. Anal. calcd. for $\mathrm{C}_{12} \mathrm{H}_{15} \mathrm{~N}_{5} \mathrm{O} \cdot 1 / 6 \mathrm{H}_{2} \mathrm{O}$ : C, 58.05; H, 6.22; N, 28.21 . Found: C, 58.36; H, 6.16; N, 27.95\%.

\section{1-(4-Benzylpiperazin-1-yl)pyrido[3,4-d]pyridazin-4(3H)- one (10)}

Reaction time: $30 \mathrm{~min}$. The crude product was purified by column chromatography using $n$-hexane-ethyl acetate gradient system. The collected product was crystallized from ethanol to yield $45.13 \%$; $\mathrm{mp} 230^{\circ} \mathrm{C}$; FTIR-ATR: 3200-2300 (N-H), $1672 \quad(\mathrm{C}=\mathrm{O}) \quad \mathrm{cm}^{-1} ; \quad{ }^{1} \mathrm{H} \quad \mathrm{NMR}$ $\left(\mathrm{DMSO}-d_{6}\right): \delta 12.37(1 \mathrm{H}, \mathrm{s}, \mathrm{NH}), 9.38(1 \mathrm{H}, \mathrm{s}, \mathrm{H} 5), 8.98$ $\left(1 \mathrm{H}, \mathrm{d}, J_{7-8}=5.6 \mathrm{~Hz}, \mathrm{H} 7\right), 7.71\left(1 \mathrm{H}, \mathrm{dd}, J_{8-7}=5.4 \mathrm{~Hz}, J_{8-5}=\right.$ $0.4 \mathrm{~Hz}, \mathrm{H} 8), 7.32-7.23\left(5 \mathrm{H}, \mathrm{m}, \mathrm{C}_{6} \mathrm{H}_{5}\right), 3.54\left(2 \mathrm{H}, \mathrm{s}, \mathrm{CH}_{2}\right)$, 3.07 (4H, m, piperazine $\mathrm{H} 2, \mathrm{H} 6), 2.58(4 \mathrm{H}, \mathrm{m}$, piperazine $\mathrm{H} 3, \mathrm{H} 5)$; HRMS calcd. for $\mathrm{C}_{18} \mathrm{H}_{20} \mathrm{~N}_{5} \mathrm{O}[\mathrm{M}-\mathrm{H}]^{+}: 322.1668$. Found 322.1666. Anal. calcd. for $\mathrm{C}_{18} \mathrm{H}_{19} \mathrm{~N}_{5} \mathrm{O}: \mathrm{C}, 67.27 ; \mathrm{H}$, 5.96; N, 21.79. Found: C, 67.31; H, 6.14; N, 21.77\%.

\section{1-(4-Methylpiperidin-1-yl)pyrido[3,4-d]pyridazin-4(3H) -one (11)}

Reaction time: $45 \mathrm{~min}$. The crude product was purified by column chromatography using $n$-hexane-ethyl ac- 
etate gradient system. Yield: $14 \%$; mp $186{ }^{\circ} \mathrm{C}$; FTIR-ATR: 3300-2700 (N-H), $1673 \quad(\mathrm{C}=\mathrm{O}) \quad \mathrm{cm}^{-1} ;{ }^{1} \mathrm{H} \quad \mathrm{NMR}$ (DMSO- $\left.d_{6}\right): \delta 12.36(1 \mathrm{H}, \mathrm{s}, \mathrm{NH}), 9.41(1 \mathrm{H}, \mathrm{s}, \mathrm{H} 5), 9.01$ $\left(1 \mathrm{H}, \mathrm{d}, J_{7-8}=5.2 \mathrm{~Hz}, \mathrm{H} 7\right), 7.71\left(1 \mathrm{H}, \mathrm{d}, J_{8-7}=5.2 \mathrm{~Hz}, \mathrm{H} 8\right)$, 3.42-3.39 (2H, m, piperidine $\mathrm{H} 2, \mathrm{H} 6), 2.74-2.69$ (2H, m, piperidine $\mathrm{H} 2, \mathrm{H} 6), 1.75-1.72(2 \mathrm{H}, \mathrm{m}$, piperidine $\mathrm{H} 3, \mathrm{H} 5)$, 1.56-1.54 $(1 \mathrm{H}, \mathrm{m}$, piperidine $\mathrm{H} 4), 1.45-1.36(2 \mathrm{H}, \mathrm{m}$, piperidine $\mathrm{H} 3, \mathrm{H} 5), 0.99\left(3 \mathrm{H}, \mathrm{d}, \mathrm{CH}_{3}\right)$; HRMS calcd. for $\mathrm{C}_{13} \mathrm{H}_{17} \mathrm{~N}_{4} \mathrm{O}[\mathrm{M}-\mathrm{H}]^{+}: 245.1402$. Found 245.1406. Anal. calcd. for $\mathrm{C}_{13} \mathrm{H}_{16} \mathrm{~N}_{4} \mathrm{O} \cdot 1 / 6 \mathrm{H}_{2} \mathrm{O}: \mathrm{C}, 63.14 ; \mathrm{H}, 6.66 ; \mathrm{N}, 22.66$. Found: C, 63.44; H, 6.61; N, 22.25\%.

\section{1-(4-Benzylpiperidin-1-yl)pyrido[3,4-d]pyridazin-4(3H)- one (12)}

Reaction time: $30 \mathrm{~min}$. The crude product was purified by column chromatography using $n$-hexane-ethyl acetate gradient system. The collected product was crystallized from methanol-water to yield $23.51 \%$; mp $147^{\circ} \mathrm{C}$; FTIR-ATR: $3200-2700(\mathrm{~N}-\mathrm{H}), 1660(\mathrm{C}=\mathrm{O}) \mathrm{cm}^{-1} ;{ }^{1} \mathrm{H}$ NMR (DMSO- $\left.d_{6}\right): \delta 12.35(1 \mathrm{H}, \mathrm{s}, \mathrm{NH}), 9.41(1 \mathrm{H}, \mathrm{s}, \mathrm{H} 5)$, $9.01\left(1 \mathrm{H}, \mathrm{d}, J_{7-8}=5.2 \mathrm{~Hz}, \mathrm{H} 7\right), 7.71\left(1 \mathrm{H}, \mathrm{d}, J_{8-7}=5.6 \mathrm{~Hz}\right.$, $\mathrm{H} 8), 7.32-7.19\left(5 \mathrm{H}, \mathrm{m}, \mathrm{C}_{6} \mathrm{H}_{5}\right), 3.44-3.39(2 \mathrm{H}, \mathrm{m}$, piperidine $\mathrm{H} 2, \mathrm{H} 6), 2.69-2.64(2 \mathrm{H}$, m, piperidine $\mathrm{H} 2, \mathrm{H} 6), 2.60$ $\left(2 \mathrm{H}, \mathrm{d}, \mathrm{CH}_{2}\right), 1.71-1.68(3 \mathrm{H}, \mathrm{m}$, piperidine $\mathrm{H} 3, \mathrm{H} 5, \mathrm{H} 4)$, 1.51-1.46 (2H, m, piperidine H3, H5); HRMS calcd. for $\mathrm{C}_{19} \mathrm{H}_{21} \mathrm{~N}_{4} \mathrm{O}[\mathrm{M}-\mathrm{H}]^{+}: 321.1715$. Found 321.1719. Anal. calcd. for $\mathrm{C}_{19} \mathrm{H}_{20} \mathrm{~N}_{4} \mathrm{O} \cdot 1 / 5 \mathrm{H}_{2} \mathrm{O}: \mathrm{C}, 70.43 ; \mathrm{H}, 6.35 ; \mathrm{N}, 17.29$. Found: C, 70.66; H, 6.25; N, 17.09\%.

\section{2. Biological Activity}

\section{2. 1. Antibacterial and Antifungal Activity ${ }^{18,19}$ Microorganisms}

Standard strains of Escherichia coli ATCC 35218, Pseudomonas aeruginosa ATCC 10145, Staphylococcus aureus ATCC 6538, Enterococcus faecalis ATCC 29212, Candida albicans ATCC 10231 and clinical isolates from Trakya University Health Center for Medical Research and Practice Microbiology Laboratory were included in the study.

\section{Microdilution Method}

Mueller Hinton Agar (MHA), Mueller Hinton Broth (MHB), Sabouraud Dextrose Agar (SDA), Sabouraud Liquid Medium (SLM) and RPMI-1640 medium with L-glutamine (Sigma) buffered with MOPS (Sigma) ( $\mathrm{pH} 7$ ) were used in the study. MHA, MHB, SDA and SLM were sterilized with autoclave at $121^{\circ} \mathrm{C}$ for 15-20 minutes and RPMI1640 was sterilized by filtration. Susceptibility testing was performed according to the guidelines of Clinical and Laboratory Standards Institute (CLSI) M100-S18 and M27-A3. $100 \mu \mathrm{L}$ of MHB and RPMI-1640 medium with L-glutamine (Sigma) buffered with MOPS ( $\mathrm{pH} 7$ ) were added to each well of the microplates for bacteria and fungi, respectively. The bacterial suspensions used for inoculation were prepared at $10^{5} \mathrm{CFU} / \mathrm{mL}$ by diluting fresh cultures at McFarland 0.5 density. Suspensions of the yeast at McFarland den- sity was diluted $1: 100$ and 1:20 respectively and $2.5 \cdot 10^{3}$ $\mathrm{CFU} / \mathrm{mL}$ were inoculated to the two fold-diluted solutions of the compounds. Stock solutions of the tested compounds were dissolved in DMSO. Standard antibiotic solutions were dissolved in appropriate solvents recommended by CLSI guidelines. Stock solutions of the tested compounds and standard drugs were diluted two-fold in the wells of the microplates so the solution of the synthesized compounds and standard drugs were prepared at 1024, 512, 256, 128, 64, 32, $16,8,4,2,1,0.5 \mu \mathrm{g} / \mathrm{mL}$ and standard drugs were prepared at $64,32,16,8,4,2,1,0.5,0.25,0.125,0.0625,0.03125 \mu \mathrm{g} / \mathrm{mL}$ concentrations. All solvents and diluents, pure microorganisms and pure media were used in control wells. A $10 \mu \mathrm{L}$ microorganisms inoculum was added to each well of the microplates. Microplates including bacteria were incubated at $37^{\circ} \mathrm{C}$ for $16-20$ hours and microplates including fungi were incubated at $35^{\circ} \mathrm{C}$ for $24-48$ hours. After incubation, the lowest concentration of the compounds that completely inhibits macroscopic growth was determined and reported as minimum inhibitory concentrations (MICs).

\section{2. 2. Antimycobacterial Activity ${ }^{20,21}$}

\section{Agar Proportion Method}

The minimum inhibitory concentration (MIC) values of each synthesized compound were tested by agar dilution in duplicate as recommended by the Clinical Laboratory Standards Institute (CLSI). ${ }^{20,21}$ Positive and negative growth controls were run in each assay. Isoniazid (INH) (Sigma I3377) and rifampicin (RIF) (Sigma R3501) were used as control agents. M. tuberculosis $\mathrm{H} 37 \mathrm{Rv}$ was used as the standard strain and was provided by Refik Saydam National Public Health Agency, National Tuberculosis Reference Laboratory, Ankara, Turkey. Stock solutions of synthesized compounds and reference compounds were prepared in $\mathrm{DMSO} / \mathrm{H}_{2} \mathrm{O}(50 \%)$ at a concentration of $1000 \mu \mathrm{g} / \mathrm{mL}$. These solutions were then filtered through a $0.22 \mu \mathrm{m}$ membrane filter (Millipore, USA). Middlebrook 7H10 agar medium (BBL, Becton Dickinson and Company, Sparks, MD, USA) was supplemented with oleic acid-albumin-dextrose-catalase (OADC, BBL, Becton Dickinson and Company, Sparks, MD, USA). Synthesized compounds and control agents were added to obtain an appropriate final concentration in the medium. The final concentrations of INH and RIF were $0.2-1 \mu \mathrm{g} / \mathrm{mL}$ and $1 \mu \mathrm{g} / \mathrm{mL}$, respectively. Synthesized compounds were prepared at final concentrations of 5, 10, 20, 40 and $80 \mu \mathrm{g} / \mathrm{mL}$. Agar without any references and synthesized compounds were used as a positive growth control, and 3 $\mathrm{mL}$ of prepared medium was dispensed into sterile tubes. The DMSO concentration in the final solutions was not above $1 \%$ for antimycobacterial activity.

\section{Inoculum Preparation}

H37Rv was maintained in Lowenstein-Jensen medium. A culture suspension was prepared by subculturing in Middlebrook 7H9 broth (BBL, Becton Dickinson and 
Company, Sparks, MD, USA) supplemented with $10 \%$ OADC at $37^{\circ} \mathrm{C}$ for $7-10$ days, until a density corresponding to $10^{-2}$ to $10^{-4}$ dilutions were obtained from McFarland standard No. 1. Then $0.1 \mathrm{~mL}$ of the diluted suspension was inoculated onto the control and the other tubes with compounds in different concentrations. The tubes were incubated at $37{ }^{\circ} \mathrm{C}$ in an atmosphere of $5 \% \mathrm{CO}_{2}$ for 3 weeks. The MIC values were defined as the lowest concentration that inhibited more than $90 \%$ of the bacterial growth and the results of INH and RIF were interpreted according to the CLSI. The MIC was considered the lowest concentration that showed no visible colonies in all dilutions.

\section{Result and Discussion}

The synthetic routes for the synthesized compounds are outlined in Scheme 1. The starting compound 2,3-dihydropyrido[3,4-d]pyridazin-1,4-dione (1) was readily prepared by the reaction of 3,4-pyridinedicarboxylic acid<smiles>O=C(O)c1ccncc1C(=O)O</smiles><smiles>CCCCCCC</smiles><smiles>O=c1[nH][nH]c(=O)c2cnccc12</smiles><smiles>CC#CC</smiles>

Compound 1<smiles>Clc1nnc(Cl)c2cnccc12</smiles><smiles>C1CCCC1</smiles>

Compound 2<smiles>[V][V]</smiles><smiles>O=c1[nH]nc(Cl)c2cnccc12</smiles>

Compound 3

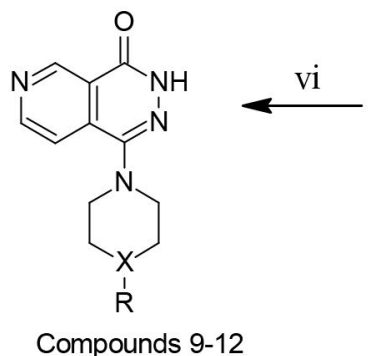

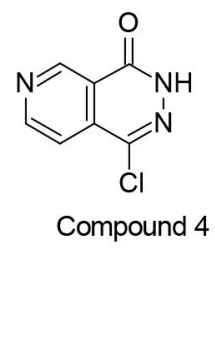

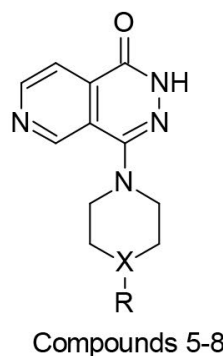

Scheme 1. Reagents and conditions: (i) acetic acid anhydride, reflux, $1 \mathrm{~h}$; (ii) hydrazine hydrate, reflux, $4 \mathrm{~h}$; (iii) phosphorus oxychloride, pyridine, reflux, 5 h; (iv) dil. $\mathrm{HCl}$, reflux, $2 \mathrm{~h}$; (v) dil. $\mathrm{HCl}$, reflux, $2 \mathrm{~h}$, neutralized with $\mathrm{NH}_{4} \mathrm{OH}$; (vi) appropriate piperazine or piperidine derivatives, diethylene glycol, and heat under MW. X = N, C; R: methyl, benzyl.

Table 1. Antibacterial, antifungal and antimycobacterial activity of the synthesized compounds

\begin{tabular}{|c|c|c|c|c|c|c|c|c|c|c|c|c|}
\hline Comp. & $\mathbf{A}$ & B & $\mathrm{C}$ & D & $\mathbf{E}$ & $\mathbf{F}$ & G & $\mathbf{H}$ & I & $\mathbf{J}$ & $\mathbf{K}$ & $\mathbf{L}$ \\
\hline 1 & 256 & 128 & 256 & 128 & 256 & 512 & 256 & 256 & 128 & 128 & 80 & 40 \\
\hline 3 & 256 & 128 & 256 & 128 & 256 & 512 & 256 & 256 & 128 & 128 & 80 & 80 \\
\hline 4 & 256 & 128 & 256 & 128 & 256 & 512 & 256 & 256 & 128 & 128 & 80 & 40 \\
\hline 5 & 256 & 256 & 256 & 128 & 256 & 512 & 256 & 256 & 128 & 128 & 80 & 40 \\
\hline 6 & 256 & 256 & 256 & 128 & 256 & 512 & 256 & 256 & 128 & 128 & 80 & 40 \\
\hline 7 & 256 & 256 & 256 & 128 & 256 & 512 & 256 & 256 & 128 & 128 & 80 & 40 \\
\hline 8 & 256 & 128 & 256 & 128 & 256 & 512 & 256 & 256 & 64 & 128 & 80 & 80 \\
\hline 9 & 256 & 128 & 256 & 128 & 256 & 256 & 256 & 256 & 128 & 128 & 80 & 40 \\
\hline 10 & 256 & 128 & 256 & 128 & 256 & 256 & 256 & 256 & 128 & 128 & 40 & 40 \\
\hline 11 & 256 & 256 & 256 & 128 & 256 & 512 & 256 & 256 & 128 & 128 & 80 & 80 \\
\hline 12 & 256 & 256 & 256 & 128 & 256 & 512 & 256 & 256 & 128 & 128 & 40 & 40 \\
\hline St1 & 2 & $<0.0078$ & 1 & 2 & 1 & 0.03125 & 1 & 1 & - & - & - & - \\
\hline St2 & 0.5 & 4 & - & - & 0.5 & 0.5 & 0.5 & 0.5 & - & - & - & - \\
\hline St3 & $>32$ & 4 & - & - & 8 & 1 & - & - & - & - & - & - \\
\hline St 4 & - & - & - & - & - & - & - & - & 1 & 1 & - & - \\
\hline St5 & - & - & - & - & - & - & - & - & - & - & 0.2 & 0.2 \\
\hline St6 & - & - & - & - & - & - & - & - & - & - & 1 & 1 \\
\hline
\end{tabular}

A: Escherichia coli ATCC 35218, B: E. coli isolate, C: Pseudomonas aeruginosa ATCC 10145, D: P. aeruginosa isolate, E: Staphylococcus aureus ATCC 6538, F: S. aureus isolate, G: Enterococcus faecalis ATCC 29212, H: E. faecalis isolate, I: Candida albicans ATCC 10231, J: C. albicans isolate, K: 10"2 Dilution of M. tuberculosis H37Rv L: 10-4 Dilution of M. tuberculosis H37Rv. St1: Meropenem, St2: Amoxicillin clavulanic acid, St3: Cefuroxime, St4: Fluconazole, St5: Isoniazid, St6: Rifampicin. 
and acetic acid anhydride and subsequent treatment with hydrazine hydrate. Compound $\mathbf{1}$ was then reacted with phosphorus oxychloride in the presence of pyridine to obtain 1,4-dichloropyrido[3,4- $d$ ] pyridazine (2). Subsequent hydrolysis under acid conditions afforded 4-chloropyrido[3,4-d]pyridazin-1(2H)-one (3). The remaining reaction medium was neutralized with ammonium hydroxide to precipitate the 1-chloropyrido[3,4- $d]$ pyridazin-4 $(3 H)$ one (4). 4-Substituted pyrido[3,4-d]pyridazin-1(2H)-one derivatives or 1-substituted pyrido[3,4- $d]$ pyridazin- $4(3 H)$ -one derivatives was readily prepared by the reaction of 4-chloropyrido[3,4- $d]$ pyridazin-1(2H)-one or 1-chloropyrido $[3,4-d]$ pyridazin-4(3H)-one and appropriate piperazine/piperidine derivatives in diethylene glycol under microwave irradiation (MW). The structures of the compounds were elucidated by FT-IR, ${ }^{1} \mathrm{H}$ NMR spectral data, HRMS and elemental analysis.

All the synthesized compounds were screened for their antimycobacterial activities against Mycobacterium tuberculosis $\mathrm{H} 37 \mathrm{Rv}$; for their antibacterial activities against for Escherichia coli ATCC 35218, Pseudomonas aeruginosa ATCC 10145, Staphylococcus aureus ATCC 6538, Enterococcus faecalis ATCC 29212 and their clinical isolates; for their antifungal activities against Candida albicans ATCC 10231 and their clinical isolates. The preliminary screening results of the prepared compounds are shown in Table 1. Results are expressed as minimal inhibitory concentration (MIC, $\mu \mathrm{g} / \mathrm{mL}$ ).

Among the synthesized compounds, compounds $\mathbf{1 0}$ and 12 displayed moderate inhibition activity against $M y$ cobacterium tuberculosis with $40 \mu \mathrm{g} / \mathrm{mL}$ MIC value $\left(10^{-2}\right.$ dilution). In $10^{-4}$ dilution, all tested compounds, except compounds 3, 8 and 11 showed moderate antitubercular activity with a MIC value of $40 \mu \mathrm{g} / \mathrm{mL}$. Besides, all compounds were found ineffective against tested Gram-positive, Gram-negative bacteria and fungus. Only compound 8 showed poor activity (MIC $64 \mu \mathrm{g} / \mathrm{mL}$ ) against Candida albicans ATCC 10231.

\section{Conclusion}

In conclusion, a series of novel pyrido[3,4- $d]$ pyridazine derivatives were designed, synthesized, and evaluated for their in vitro antimycobacterial activities against $M y$ cobacterium tuberculosis H37Rv. Among the synthesized compounds, compounds $\mathbf{1 0}$ and $\mathbf{1 2}$ exhibited promising antimycobacterial activity with MIC value of $40 \mu \mathrm{g} / \mathrm{mL}$.

Conflict of Interest. The authors declare that they have no conflict of interest.

\section{References}

1. Global Tuberculosis Report 2017, Geneva, World Health Organization (WHO), 2017.
2. Guidelines for Treatment of Drug-susceptible Tuberculosis and Patient Care, 2017 Update. Geneva, World Health Organization (WHO), 2017.

3. WHO Treatment Guidelines for Drug-Resistant Tuberculosis, 2016 Update. Geneva, World Health Organization (WHO), 2016.

4. M. Stehr, A. A. Elamin, M. Singh, Expert Rev. Anti Infect. Ther. 2015, 13, 593-603.

DOI:10.1586/14787210.2015.1021784

5. L. A. Dutra, T. R. F. deMelo, C. M. J. L. Chin dos Santos, Int. Res. J. Pharm. Pharmacol. 2012, 2, 1-9.

6. H. H. Jardosh, M. P. Patel, Eur. J. Med. Chem. 2013, 65, 348359. DOI:10.1016/j.ejmech.2013.05.003

7. N. Anand, K. Upadhyaya, R. P. Tripathi, Chemistry and Biology Interface, 2015, 5, 84-127.

8. G. F. dos Santos Fernandes, P. C. de Souza, E. Moreno-Viguri, M. Santivañez-Veliz, R. Paucar, S. Pérez-Silanes, K. Chegaev, S. Guglielmo, L. Lazzarato, R. Fruttero, C. Man Chin, P. B. da Silva, M. Chorilli, M. C. Solcia, C. M. Ribeiro, C. S. P. Silva, L. B. Marino, P. L. Bosquesi, D. M. Hunt, L. P. S. de Carvalho, C. A. de Souza Costa, S. H. Cho, Y. Wang, S. G. Franzblau, F. R. Pavan, J. L. dos Santos, J. Med. Chem. 2017, 60, 8647-8660. DOI:10.1021/acs.jmedchem.7b01332

9. F. S. Castelo-Branco, E. C. de Lima, J. L. de Oliveira Domingos, A. C. Pinto, M. C. S. Lourenço, K. M. Gomes, M. M. Costa-Lima, C. F. Araujo-Lima, C. A. F. Aiub, I. Felzenszwalb, T. E. M. M. Costa, C. Penido, M. G. Henriques, N. Boechat, Eur. J. Med. Chem. 2018, 146, 529-540.

DOI:10.1016/j.ejmech.2018.01.071

10. J. Zitko, P. Paterová, V. Kubíček, J. Mandíková, F. Trejtnar, J. Kuneš, M. Doležal, Bioorg. Med. Chem. Lett. 2013, 23, 476479. DOI:10.1016/j.bmcl.2012.11.052

11. B. Servusová, P. Paterová, J. Mandíková, V. Kubíček, R. Kučear, J. Kuneš, M. Doležal, J. Zitko, Bioorg. Med. Chem. Lett. 2014, 24, 450-453. DOI:10.1016/j.bmcl.2013.12.054

12. Y.-Q. Hu, S. Zhang, F. Zhao, C. Gao, L.-S. Feng, Z.-S. Lv, Z. Xu, X. Wu, Eur. J. Med. Chem. 2017, 133, 255-267.

DOI:10.1016/j.ejmech.2017.04.002

13. I. Matsuura, K. Okui, Chem. Pharm. Bull. 1969, 17, 22662272. DOI:10.1248/cpb.17.2266

14. K. Körmendy, T. Kovács, F. Ruff, I. Kövesdi, Acta Chim. Hung. 1983, 112, 487-499.

15. J. A. Kaizerman, W. Aaron, S. An, R. Austin, M. Brown, A. Chong, T. Huang, R. Hungate, B. Jiang, M. G. Johnson, G. Lee, B. S. Lucas, J. Orf, M. Rong, M. M. Toteva, D. Wickramasinghe, G. Xu, Q. Ye, W. Zhong, D. L. McMinn, Bioorg. Med. Chem. Lett., 2010, 20, 4607-4610.

DOI:10.1016/j.bmcl.2010.06.006

16. J. R. Greenwood: Pyridazinediones and Amino Acid Receptors: Theoretical Studies, Design, Synthesis, and Evaluation of Novel Analogues, PhD Thesis, The Department of Pharmacology, The University of Sydney. 1999, pp. 1-262.

17. B. Prek, B. Stanovnik, Acta Chim. Slov. 2017, 64, 798-803. DOI:10.17344/acsi.2017.3695

18. Clinical and Laboratory Standards Institute (CLSI) (formerly NCCLS). Performance Standards for Antimicrobial 
Susceptibility Testing $18^{\text {th }}$ Informational Supplement. CLSI M100-S18, Clinical and Laboratory Standards Institute, 940 West Valley Road, Wayne, Pennsylvania, USA, 2008.

19. Clinical and Laboratory Standards Institute (CLSI) (formerly NCCLS). Reference Method for Broth Dilution Antifungal Susceptibility Testing of Yeast Approved Standard, M27-A3, Clinical and Laboratory Standards Institute, 940 West Valley Road, Wayne, Pennsylvania, USA, 2006.
20. National Committee for Clinical Laboratory Standards. Susceptibility Testing of Mycobacteria, Nocardia, and Other Aerobic Actinomycetes: Approved Standard NCCLS Document M24-A. Wayne, Pennsylvania, 2003.

21. Clinical and Laboratory Standards Institute (CLSI) (formerly NCCLS). Antimycobacterial Susceptibility Testing for M. tuberculosis: Tentative Standard NCCLS Document M24-T. Villanova, Pennsylvania, 2002.

\section{Povzetek}

$\mathrm{V}$ tej študiji smo sintetizirali nekaj novih pirido[3,4-d]piridazinskih derivatov in ovrednotili njihove in vitro protibakterijske, protiglivične in antimikobakterijske aktivnosti. Med sintetiziranimi spojinami sta spojini 10 (1-(4-benzilpiperazin-1-il)pirido[3,4-d]piridazin-4(3H)-on) in 12 (1-(4-benzilpiperidin-1-il)pirido[3,4-d]piridazin-4(3H)-on) izkazali največjo antimikobakterijsko aktivnost. Vendar so se vse spojine izkazale kot neučinkovite proti testiranim Gram-pozitivnim in Gram-negativnim bakterijam ter glivam. 